\title{
Induction Therapy for Patients with Multiple Sclerosis: Why? When? How?
}

\author{
Gilles Edan · Emmanuelle Le Page
}

Published online: 9 May 2013

(c) The Author(s) 2013. This article is published with open access at Springerlink.com

\begin{abstract}
The concept of induction treatment followed by long-term maintenance treatment in multiple sclerosis (MS) has attracted considerable attention. The combination of mitoxantrone as the induction therapy followed by an immunomodulatory drug (e.g., interferon beta or glatiramer acetate) as the maintenance therapy is of particular interest. This approach is suitable for patients with particularly aggressive disease, characterised by frequent relapses with incomplete recovery and the accumulation of focal lesions visible on magnetic resonance imaging. A long-term study has shown that a short (6 month) course of mitoxantrone followed by maintenance therapy with an immunomodulatory drug brings about a rapid reduction in disease activity and subsequent sustained disease control for at least 5 years. Furthermore, randomised studies have demonstrated that induction with mitoxantrone followed by maintenance treatment affords better disease control than monotherapy with an interferon beta. Natalizumab is also effective in patients with very active MS, but has a propensity to result in rebound inflammatory disease activity on withdrawal. More recently, a mere 5-day course of 12-mg intravenous perfusions of alemtuzumab was found to bring long-term clinical benefits in early relapsing MS patients at risk of developing severe systemic autoimmune disease within the space of a few years.
\end{abstract}

\section{Induction Therapy in Multiple Sclerosis: Why?}

The concept of induction treatment with one drug followed by long-term maintenance treatment with a different drug

G. Edan $(\bowtie) \cdot$ E. Le Page

Department of Neurology, CIC-P 02-03 INSERM, INCR,

CHU Pontchaillou, 35033 Rennes Cedex, France

e-mail: gilles.edan@chu-rennes.fr has a proven utility in oncology [1]. In multiple sclerosis (MS), induction therapy seems to bring both short- and long-lasting benefits. From a theoretical point of view, there are two contrasting treatment regimens in $\operatorname{MS}[2,3]$ : induction versus escalating. The rationale behind escalating therapy is that treatment starts with safe drugs and only moves on to more aggressive ones if the ongoing treatment fails. In the escalating approach, glatiramer acetate and interferon betas are regarded as first-line drugs, immunosuppressants (mitoxantrone, natalizumab, fingolimod, alemtuzumab) as second-line ones, and very intensive immunosuppression (autologous bone marrow transplantation, high-dose cyclophosphamide) as third-line ones. The key to the success of escalation therapy is to define upfront with the patient the exact suboptimal response threshold at which the next-level therapeutic option should be introduced. The decision to adopt a second-line therapy in patients who respond poorly to first-line therapy should not be delayed until severe and irreversible disability has set in. Given that all the immunosuppressants that are currently available present potentially serious side effects, the induction strategy has generally been reserved for patients with very active and aggressive disease. In these patients, there is an acknowledged risk of early disability, and once neurological function is lost, it cannot be regained. In such patients, this disease-inherent risk can be assumed to outweigh that associated with the use of powerful immunosuppressants. This treatment strategy involves the use of immunosuppressants for the minimum amount of time needed to gain adequate control over disease activity. Once disease control has been achieved, treatment can be switched to maintenance therapy with a better tolerated drug. This approach may be a useful and conservative means of using these highly effective therapies whilst minimising exposure and the attendant safety risk. 


\section{Induction Therapy in Multiple Sclerosis: When?}

Most of the consensus groups support an early start to treatment in patients with a diagnosis of MS. One major goal of treatment is to prevent the accumulation of irreversible neurological disability and, in particular, to prevent conversion to a secondary progressive course. Current immunotherapies cannot be expected to be effective once conversion has occurred as by then the focal inflammatory process is less relevant. Indeed, randomised clinical trials have indicated that such treatments are largely, if not totally, ineffectual in secondary progressive disease $[4,5]$. Epidemiological data suggest that the disability progression in MS takes place in two stages, the first stage being dependent on focal central nervous system (CNS) inflammatory lesions and the second stage being independent of focal CNS inflammatory lesions [6]. Patients who experience frequent relapses in the early stage of disease and those who accumulate a large number of focal lesions visible on 2-T (T2) magnetic resonance imaging (MRI) within the first 5 years become handicapped more quickly than those patients who do not [6-8]. This is probably because the nature of the immune disturbance characterising MS changes over time, probably shifting from a peripheral immunopathological profile to a compartmentalised CNS profile in late MS [9]. As the target of all currently approved treatments is focal CNS inflammation, which predominates at the beginning of the disease course, it is all the more logical to concentrate therapeutic efforts on the early phase of the disease. The "induction treatment strategy" deserves consideration when the patient responds to the clinical and MRI criteria defined in Table 1, having received a first line treatment with immunomodulatory drugs or not.

\section{Induction Therapy in Multiple Sclerosis: How?}

In MS, induction has been investigated in protocols using mitoxantrone, natalizumab, and alemtuzumab (Table 2).

Table 1 Clinical and MRI definition of aggressive RRMS suitable for an induction strategy

\section{Pure RRMS}

2. Age $<40$ years

3. Highly active disease with at least 2 or more relapses within the previous 12 months

4. Severe relapse resulting in EDSS score $\geq 4$

5. Worsening EDSS score due to relapses (increase of 2 or more points within the previous 12 months)

6. Two or more additional Gd+ lesions on recent T2 MRI

RRMS relapsing remitting multiple sclerosis, EDSS Expanded Disability Status Scale
Mitoxantrone was first studied in induction protocols in relapsing remitting MS (RRMS) as for at least 10 years it was the only immunosuppressant to be approved for this indication in most countries. Natalizumab is also effective in patients with very active MS, but has the propensity to result in rebound inflammatory disease activity on withdrawal [8]. A mere 5-day course of alemtuzumab administered intravenously ( $12 \mathrm{mg}$ ) was recently demonstrated to bring a long-term clinical benefit in early relapsing MS.

\section{Mitoxantrone}

\subsection{Mitoxantrone as Induction: The Pivotal Trial}

A randomised clinical trial [10] assessed the short-term benefits of mitoxantrone treatment for 6 months in 42 patients with very active RRMS or secondary progressive multiple sclerosis (SPMS), defined as the occurrence of two or more relapses without recovery or disability progression by more than two points on the EDSS (Expanded Disability Status Scale) within the previous 12 months together with MRI evidence of active disease. Patients were randomised to receive either mitoxantrone $(20 \mathrm{mg}$ IV) and methylprednisolone (1 g IV) every month or methylprednisolone alone over a 6-month treatment period. In the mitoxantrone group (Table 2), the proportion of patients who did not develop new $\mathrm{Gd}+$ lesions $(90 \%)$ was significantly higher than it was in the control group (31\%). In the mitoxantrone group, $66 \%$ of the patients remained relapse-free during the 6-month treatment period compared with $33 \%$ in the control group. Similarly, with respect to disability progression, 12 out of 21 patients in the mitoxantrone group improved by at least one point on the EDSS and only one deteriorated. By contrast, in the control group, six deteriorated and only three improved. The rapid and powerful impact of mitoxantrone encouraged its use as an induction treatment in MS.

\subsection{Mitoxantrone as Induction: An Observational 5-Year Follow-Up Study}

An observational study (Table 2) was performed in our centre, prospectively assessing 100 consecutive very active relapsing MS patients over a 5-year period using the same administration regimen as before [11]. The majority of patients $(73 \%)$ were assigned to a maintenance treatment following induction with mitoxantrone. In the year preceding treatment, the annualised relapse rate in the patient group was 3.3, and the EDSS score progressed by a mean of 2.2 points. In addition, $\mathrm{Gd}+$ lesions were visible on MRI for $84 \%$ of the sample. These patients thus presented very active disease. 


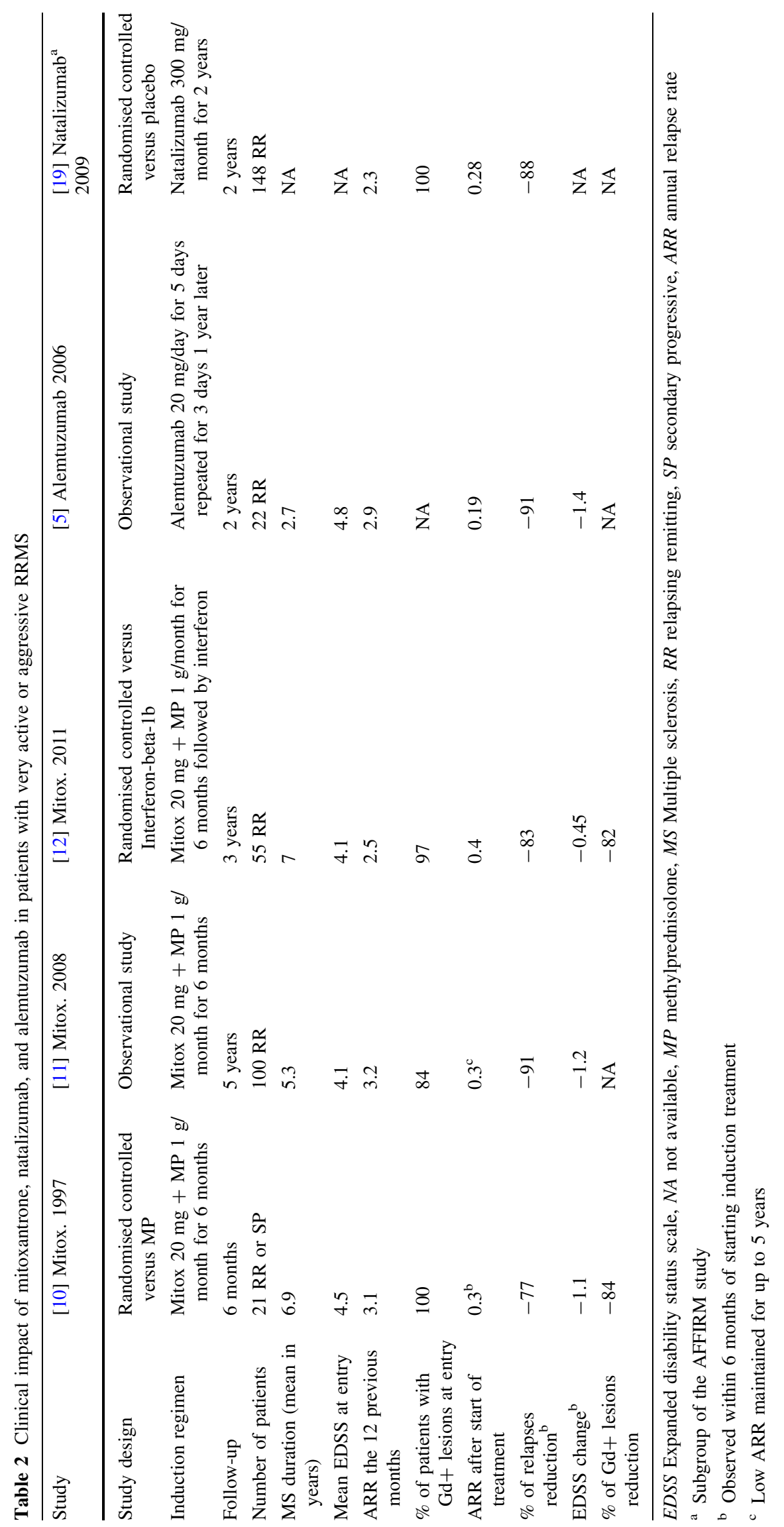


In the first year following the initial administration of mitoxantrone, the relapse rate declined to 0.29 relapses per year-a reduction of $91 \%$. This reduction was maintained across the 5-year observation period, oscillating between 0.3 and 0.4 relapses/year. Around one third of patients remained free of relapses throughout the 5-year period, and the median time to first relapse was 2.72 years. Likewise, the proportion of patients whose disability deteriorated by at least one point on the EDSS, confirmed at 3 months, decreased from $88 \%$ in the year preceding treatment to $5 \%$ for the first year. Furthermore, the clinical benefit at 1 year in terms of disability was maintained in $59 \%$ of patients throughout the 5-year observation period.

Potential determinants of a good treatment response were assessed. The five patients whose disability worsened during the first year of treatment were significantly $(p<0.02)$ older when treatment was started (41 years) than those whose disability stabilised or improved (32 years). The 20 patients who converted to SPMS during the follow-up period were significantly $(p<0.02)$ more disabled (mean EDSS score 4.8) at the start of treatment than those who did not (mean EDSS score 3.9).

\subsection{Mitoxantrone as Induction Followed by a Maintenance Therapy with Interferon $\beta$}

This randomised controlled trial [12] included patients with RRMS who had experienced at least two relapses with incomplete recovery the previous year and who displayed $\mathrm{Gd}+$ lesions on MRI. Fifty-five patients were randomly allocated to the 6-month mitoxantrone induction regimen used in the previous studies followed by a 3-month washout period, then interferon beta-1b (Table 2). The other study arm (54 patients) received interferon beta-1b for 3 years, combined with methylprednisolone at $1 \mathrm{~g}$ IV/month for the first 6 months. The two patient groups had comparable clinical and demographic features at inclusion. Patients underwent a complete neurological examination every 3 months and spin-echo MRI at inclusion and at months 9, 24, and 36 .

Compared with the interferon beta group, the time to sustained worsening of EDSS by one point was delayed in the mitoxantrone group $(p<0.012)$. The 3 -year risk of sustained worsening of disability was reduced by $65 \%$ (12 vs. $34 \%)$. Mitoxantrone delayed the time of confirmed worsening of disability by 18 months compared with the interferon beta group. The raw percentage of patients with sustained worsening of disability was also reduced (9.1 vs. $25.9 \%$ ). The mean EDSS score had improved by 0.45 point at the last observation point (M36) in the mitoxantrone group (from 4.16 to $3.66, p=0.007$ ), but remained unchanged in the interferon beta group (from 3.86 to 3.76 , $p=0.771$ ). Post hoc analyses suggested that this induction strategy using mitoxantrone is more effective in patients with a lower disability: the risk of sustained worsening of disability was reduced by $86.9 \%$ in patients with a low baseline EDSS $(<4)$. Throughout the study, from baseline to month 36 , the mitoxantrone group enjoyed a lower annualised relapse rate both per patient $(0.4$ vs. $1.1, p<0.03)$ and per group $(p<0.001)$. The time to first relapse after treatment institution was delayed in the mitoxantrone group by 21 months compared with the interferon beta group $(p<0.001)$. The proportion of patients who remained free of relapses throughout the follow-up period was $53 \%$ in the induction group and $26 \%$ in the monotherapy group $(p<0.01)$.

The mean number of new lesions on T2 MRI was significantly lower in the mitoxantrone group at each of the measurement points. The mean cumulative number of new T2 lesions over 36 months was lower in patients in the mitoxantrone group than in patients in the interferon beta group (3.6 vs. 9.8; $p=0.04$ ). The mean number of $\mathrm{Gd}+$ lesions was also lower in the mitoxantrone group at month 9 (0.36 vs. $2.1 ; p=0.012)$, and the percentage of patients without any $\mathrm{Gd}+$ lesions was higher ( 88 vs. $57 \%$ at month $9 ; p=0.010$ ).

\subsection{Mitoxantrone as Induction Followed by a Maintenance Therapy with Glatiramer [13]}

In this controlled study, 40 relapsing MS patients with 1-15 Gd+ lesions on MRI and EDSS scores of 0-6.5 were randomised to receive either short-term induction therapy with mitoxantrone (3-monthly infusions at $12 \mathrm{mg} / \mathrm{m}^{2}$ ) followed by 12 months of daily glatiramer acetate therapy at $20 \mathrm{mg} /$ day subcutaneously for a total of 15 months $(n=21)$ or else glatiramer acetate at $20 \mathrm{mg} /$ day for 15 months $(n=19)$. MRI scans were performed at months 6, 9, 12 and 15. Mitoxantrone-glatiramer acetate induction produced an $89 \%$ greater reduction [relative risk $(\mathrm{RR})=0.11, p=0.0001]$ in the number of $\mathrm{Gd}+$ lesions at months 6 and 9 than glatiramer acetate alone and a $70 \%$ greater reduction $(\mathrm{RR}=0.30, p=0.0147)$ at months 12 and 15. Mean relapse rates were 0.16 and 0.32 in the mitoxantrone-glatiramer acetate and glatiramer acetate groups. Short-term immunosuppression with mitoxantrone followed by daily glatiramer acetate for up to 15 months was found to be safe and effective, with an early and sustained decrease in MRI disease activity.

\subsection{Mitoxantrone as Induction: the Safety Profile [14]}

The year 2001 saw the launch of a French multicentre prospective study of a large cohort of MS patients with annual updates for at least the first 5 years after initiation of mitoxantrone therapy in order to determine the long-term 
safety profile of mitoxantrone in MS. A total of 802 patients from $12 \mathrm{MS}$ centres [308 RRMS, 352 SPMS and 142 primary progressive MS (PPMS)] received either monthly infusions of mitoxantrone $(87 \%)$ or else one infusion every 3 months (13\%) with a mean cumulative dose of $72 \mathrm{mg} / \mathrm{m}^{2}$. Patients underwent clinical and haematological assessments before every infusion as well as every 6-12 months up to 5 years after starting the mitoxantrone. Echocardiograms were performed at the start and end of mitoxantrone treatment and yearly up to 5 years afterwards.

The cohort was followed for 5,354 patient-years. One out of 802 patients $(0.1 \%)$ presented with acute congestive heart failure. Two cases of therapy-related leukaemia $(0.25 \%)$ were detected 20 months after starting the mitoxantrone (one death and one with 8 years' confirmed remission). Of the 317 women treated before the age of 45 , $17.3 \%$ developed persistent age-dependent amenorrhoea (5.4\% before 35 years, $30.7 \%$ after 35 years). The risk of sterility in young fertile women was lower $(0 \%$ before 25 years, $4.5 \%$ between 25 and 30 years). The only prospective study of a large cohort with at least 5 years of follow-up to be published so far, it provided good insights into the long-term safety profile of the use of mitoxantrone in an induction treatment protocol in MS patients. Other studies of the risk of acute leukaemia have been published, given similar risk [15] or higher risk [16], but they were retrospective and involved a different regimen. In men, there is no documented risk of sterility: after cessation of mitox there is generally complete recovery of sperm production without morphological changes in vitro [17].

These studies involving a mitoxantrone induction regimen in patients with very aggressive RRMS suggest that it can greatly reduce disease activity, particularly when followed by a maintenance therapy with a disease-modifying immunomodulatory drug. With the treatment protocol recommended for use in France (6-monthly IV infusions of mitoxantrone at a cumulative dose of $72 \mathrm{mg} / \mathrm{m}^{2}$ ), mitoxantrone had an a reasonable risk/benefit profile. These findings encourage the use of such induction regimens in patients who present early signs of aggressive disease.

\section{Natalizumab}

\subsection{Natalizumab in Very Active Disease}

In the AFFIRM study [18], 942 RR MS patients were randomised to receive either natalizumab $(300 \mathrm{mg})$ or placebo intravenously every 4 weeks over a 2 -year period. A post hoc analysis [19] was conducted to determine the efficacy of the natalizumab in those patients who presented with highly active disease (i.e., $\geq 2$ relapses in the year before study entry and $>1 \mathrm{Gd}+$ lesion on MRI). Within the AFFIRM sample, 148 natalizumab patients and 61 placebo patients met the criteria for highly active disease (Table 2). In this treatment-naïve AFFIRM population with highly active disease, the 2-year cumulative probability of disability progression sustained for 3 months was $14 \%$ in the natalizumab group and $29 \%$ in the placebo group $(p=0.03)$. Natalizumab reduced the cumulative probability of relapse over 2 years by $81 \%$ compared with the placebo $(0.28$ vs. $1.46, p<0.0001)$.

\subsection{Natalizumab Safety Profile}

With natalizumab, the main concern is the risk of progressive multifocal leukoencephalopathy (PML). Recent data have made it possible to stratify this risk on the basis of anti-JC antibody virus testing, duration of drug exposure, and former use of immunosuppressants [20]. After the first two years, the risk of PML is restricted to patients with prior use of immunosuppressants: one in 96 patients compared with one in 256 patients with no prior use of immunosuppressants.

Natalizumab is not an ideal candidate for induction use as it has no residual impact after withdrawal, thus exposing patients to a risk of rapid rebound [21]. While it is now clear that the therapeutic gap should be avoided, it remains unclear which alternative drugs should be used to limit this risk of rebound after natalizumab [22].

\section{Alemtuzumab}

Even if not yet licensed, alemtuzumab deserves to be considered as a good candidate for induction therapy in MS. In patients who have received alemtuzumab, cells carrying CD52 antigens (lymphocytes and monocytes) are no longer detectable in the circulation. Monocyte numbers return to normal within 3 months, and the median recovery times to baseline levels for CD4+ and CD8+ cells are 60 and 30 months. B cells return to normal within 3 months. Alemtuzumab is effective in patients with very active RRMS and is a more effective treatment than interferon betas for early RRMS.

\subsection{Alemtuzumab is Effective in Patients with Very Active RRMS}

In this observational study [5], 22 patients with a very active form of RRMS (i.e., a mean annual relapse rate of 2.9 and mean increase of 2.2 in the EDSS score within the 12 months before treatment) received five daily doses of alemtuzumab at $20 \mathrm{mg}$ administered by intravenous 
infusion over $4 \mathrm{~h}$ preceded by a 1-h infusion of methylprednisolone at $1 \mathrm{~g}$. Nineteen of the 22 patients received a second course 12-18 months later (three daily 20-mg doses).

Before they started the alemtuzumab, the patients had a mean disease duration of 2.7 years and a mean EDSS score of 4.8. Seventeen were drug-naïve patients and five were interferon beta nonresponders. Patients were assessed over a mean follow-up period of 29 months.

After treatment, the cohort had a significant $91 \%$ reduction in the relapse rate $(p<0.001)$, with an annual relapse rate of 0.19 and a marked reduction of 1.2 points in the EDSS score at month 6 and 1.4 points at months 12 and 24 (Table 2).

\subsection{Alemtuzumab Is a More Effective Treatment than Interferon $\beta$ in Patients with Early Active RRMS}

This has been clearly documented in three controlled trials [23-25], involving 334, 581, and 638 RRMS patients. In these studies, alemtuzumab was compared with interferon beta-1a (44 $\mu \mathrm{g}$ subcutaneously 3 times per week for 2 years). Sustained accumulation of disability was observed in 9,8 , and $13 \%$ of patients in the alemtuzumab groups compared with 26,11 , and $20 \%$ of patients in the interferon beta-1a groups $(p<0.001, p=0.70$, and $p=0.008)$. The annual relapse rates were lower in the alemtuzumab groups $(0.10,0.19,0.26)$ than in the interferon beta-1a groups $(0.36,0.38$, and $0.52, p<0.001$ in all three studies). The percentages of relapse-free patients were 79,78 , and $65 \%$ in the alemtuzumab groups versus 60,59 , and $47 \%$ in the interferon beta-1a groups. Used at the dose of $12 \mathrm{mg}$ intravenously per day for 5 days, with a retreatment at the same dose for 3 days 1 year later, alemtuzumab had a profound and sustained effect on the inflammatory process.

\subsection{Alemtuzumab: 5-Year Safety Profile}

The extension of the CAMMS223 trial yielded long-term safety and efficacy results $[26,27]$. The cumulative risk of autoimmune diseases at 5 years follow-up was about $22.2 \%$, thyroid Graves' disease $11.7 \%$, immune thrombocythemia purpura (ITP) $3 \%$, and severe glomerulopathy (Goodpasture's disease) $0.4 \%$. Adequate monitoring dramatically reduces the potential severity of these autoimmune diseases.

\section{Conclusion}

The current challenge in treating MS is to identify the most effective drug and strategy for each individual patient at each phase of the disease. Both escalating and induction strategies can be successfully applied on the basis of clinical and radiological tools. Apart from clinically aggressive RRMS, for which induction treatment should certainly be regarded as the first line of treatment, there is a lack of biomarkers to guide early choices between these two strategies at an individual level. In the coming years, new MRI techniques (brain and spinal cord imaging) should help us to identify those RRMS patients, especially individuals without any real disability, who are most at risk of developing destructive CNS lesions with or without firstline therapy and who are therefore more eligible for an early and more aggressive treatment strategy. We also need to assess how far the early complete control of the focal inflammatory process (no relapse, no additional MRI focal lesions) in RRMS patients stops or delays the conversion to the SPMS phenotype. Furthermore, the use of more aggressive drugs in MS will make it particularly important to have longer term safety data than are currently available for the more recent antiinflammatory drugs.

Conflict of interest GE has received honoraria from advisory board participation from Bayer, Biogen Idec, and LFB, and his institution has received support from Bayer, Merck Serono, and TEVA and from ARSEP Foundation. ELP has received compensation as a speaker from Biogen Idec, Serono, Sanofi-Aventis, and Bayer Schering Pharma AG. No sources of funding were received for writing this manuscript.

Open Access This article is distributed under the terms of the Creative Commons Attribution Noncommercial License which permits any noncommercial use, distribution, and reproduction in any medium, provided the original author(s) and the source are credited.

\section{References}

1. Pui CH, Evans WE. Treatment of acute lymphoblastic leukemia. N Engl J Med. 2006;354:166-78.

2. Comi G. Induction vs. escalating therapy in multiple sclerosis: practical implications. Neurol Sci. 2008;29:S253-5.

3. Reickmann P. Concepts of induction and escalation therapy in multiple sclerosis. J Neurol Sci. 2009;277(S1):S42-5.

4. Panitch H, Miller A, Paty D, et al. North American Study Group on interferon beta $1 \mathrm{~b}$ in secondary progressive MS: result from a 3 year controlled study. Neurology. 2004;63:1788-95.

5. Coles AJ, Cox E, Le Page E, et al. The window of therapeutic opportunity in multiple sclerosis: evidence from monoclonal antibody therapy. J Neurol. 2006;253:98-108.

6. Leray E, Yaouanq J, Le Page E, et al. Evidence of a two stage disability progression in multiple sclerosis. Brain. 2010;133: 1900-13.

7. Scalfari A, Neuhaus A, Degenhardt A, et al. The natural history of multiple sclerosis, a geographically based study. 10. Relapses and long-term disability. Brain. 2010;133:1914-29.

8. Fisniku LK, Brex PA, Altmann DR, et al. Disability and T2 MRI lesions: a 20-year follow-up of patients with relapse onset of multiple sclerosis. Brain. 2008;131:808-17.

9. Weiner HL. The challenge of multiple sclerosis: How do we cure a chronic heterogeneous disease? Ann Neurol. 2009;65:239-48. 
10. Edan G, Miller D, Clanet M, et al. Therapeutic effect of mitoxantrone combined with methylprednisolone in multiple sclerosis: a randomised multicenter study of active disease using MRI and clinical criteria. J Neurol Neurosurg Psychiatry. 1997;62:112-8

11. Le Page E, Leray E, Taurin G. etal. Mitoxantrone as induction treatment in aggressive relapsing remitting multiple sclerosis: treatment response factors in a 5 year follow-up observational study of 100 consecutive patients. J Neurol Neurosurg Psychiatry. 2008;79:52-6.

12. Edan G, Comi C, Le Page E, et al. Mitoxantrone prior to interferon beta-1b in aggressive relapsing multiple sclerosis: a 3-year randomised trial. J Neurol Neurosurg Psychiatry. 2011;82:1344-50.

13. Vollmer T, Panitch H, Bar-Or A. Glatiramer acetate after induction therapy with mitoxantrone in relapsing multiple sclerosis. Mult Scler. 2008;14(5):663-70.

14. Le Page E, Leray E, Edan G. Long-term safety profile of mitoxantrone in a French cohort of 802 multiple sclerosis patients: a 5-year prospective study. Mult Scler. 2011;17:867-75.

15. Stroet A, Hemmelmann C, Starck M, et al. Incidence of therapyrelated acute leukaemia in mitoxantrone-treated multiple sclerosis patients in Germany. Ther Adv Neurol Disord. 2012;5(2):75-9.

16. Martinelli V, Cocco E. Capra R et al Acute myeloid leukemia in Italian patients with multiple sclerosis treated with mitoxantrone. Neurology. 2011;77(21):1887-95.

17. Meistrich ML, Wilson G, Mathur K, et al. Rapid recovery of spermatogenesis after mitoxantrone, vincristine, vinblastine, and prednisone chemotherapy for Hodgkin's disease. J Clin Oncol. 1997; 15:3488-95.

18. Polman Ch, O'Connor PW, Havrdora E, et al. A randomized, placebo controlled trial of natalizumab of relapsing multiple sclerosis. N Engl J Med. 2006;354(9):899-910.
19. Hutchinson M, Kappos L, Calabresi P, et al. The efficacy of natalizumab in patients with relapsing multiple sclerosis: subgroup analyses of AFFIRM and SENTINEL. J Neurol. 2009; 256:405-15.

20. Sorensen PS, Bertolotto A, Edan G, et al. Risk stratification for progressive multiple leukoencephalopathy in patients treated with natalizumab. Mult Scler J. 2012;18(2):143-52.

21. Kerbrat A, Le Page E, Leray E, et al. Natalizumab and drug holiday in clinical practice: An observational study in very active relapsing remitting multiple sclerosis patients. J Neurol Sci. 2011;308:98-102.

22. Sempere AP, Martin-Medina P, Berenguer-Ruiz L, et al. Switching from natalizumab to fingolimod: an observational study. Acta Neurol Scand. 2013. doi:10.1111/ane.12082.

23. The CAMMS223 Trial Investigators. Alemtuzumab vs. interferon beta-1a in early multiple sclerosis. N Engl J Med. 2008;359: 1786-801.

24. Cohen JA, Coles AJ, Arnold DL, et al. Alemtuzumab versus interferon beta $1 \mathrm{a}$ as first-line treatment for patients with relapsing-remitting multiple sclerosis: a randomized controlled phase 3 trial. Lancet. 2012;380:1819-28.

25. Coles AJ, Twyman CL, Arnold DL, et al. Alemtuzumab for patients with relapsing multiple sclerosis after disease-modifying therapy: a randomized phase 3 trial. Lancet. 2012;380:1829-39.

26. Coles AJ, Cox E, Vladic A, et al. Alemtuzumab more effective than interferon $\beta$-1a at 5 -year follow-up of CAMMS223 clinical trial. Neurology. 2012;78:1069-78.

27. Cossburn M, Pace AA, Jones JJ, et al. Autoimmune disease after alemtuzumab treatment for multiple sclerosis in a multicenter cohort. Neurology. 2011;77:573-9. 